\title{
Nouveaux matériaux non linéaires pour la conversion de fréquence
}

\author{
Ch. Wyon \\ LETI (CEA-Technologies Avancées), DOPT/MDO, CEN/G, 85 X, 38041 Grenoble Cedex, \\ France
}

Generation of UV light by frequency conversion of laser beams using new nonlinear materials is presented. After a brief introduction of the basic harmonic generation mechanisms and a survey of the nonlinear optical properties of some crystals, the properties of $\mathrm{BBO}$ and $\mathrm{LBO}$ are described.

\section{INTRODUCTION}

L'intérêt pour de nouveaux matériaux non linéaires s'est accru depuis plusieurs années car la puissance délivrée par les lasers solides a considérablement augmenté. La réalisation de sources lumineuses intenses et cohérentes émettant dans le visible et l'ultraviolet devient alors envisageable.

Les principales applications des matériaux non linéaires sont :

. l'obtention de lasers verts $(532 \mathrm{~nm})$ par doublage d'un laser YAG:Nd (1064 nm) où une forte puissance moyenne est plus spécifiquement recherchée pour être utilisés en photochimie, biologie, interférométrie holographique, séparation isotopique...

. La mise au point de lasers bleus (400 nm) par génération du second harmonique des diodes laser AlGaAs dont les applications pour l'enregistrement optique nécessitent des sources compactes et peu onéreuses.

- La réalisation de sources cohérentes dans IUV par génération des harmoniques des lasers solides (YAG:Nd, $\mathrm{Al}_{2} \mathrm{O}_{3}: \mathrm{Ti}, \mathrm{BeAl}_{2} \mathrm{O}_{4}: \mathrm{Cr}$...), des lasers à $\mathrm{gaz}\left(\mathrm{Ar}^{+}, \mathrm{Cu}^{+} \ldots\right)$ ou à colorants dont les principales applications ont été développées au cours de ce colloque.

Après une description des principes généraux gouvernant la conversion de fréquence, les différents matériaux susceptibles de convenir pour la génération d'harmoniques dans l'ultraviolet seront présentés et les propriétés des deux matériaux les mieux adaptés : le $\mathrm{BBO}\left(\mathrm{\beta}: \mathrm{BaB}_{2} \mathrm{O}_{4}\right)$ et le $\mathrm{LBO}\left(\mathrm{LiB}_{3} \mathrm{O}_{5}\right)$ seront plus précisément décrites.

\section{GENERALITES SUR LA CONVERSION DE FREOUENCE}

\section{1 Principe de base}

Lorsqu'un faisceau lumineux traverse une phase condensée transparente son champ électrique interagit avec les électrons de valence des atomes ou des molécules du milieu condensé. S'il y a résonance, des phénomènes d'absorption ou d'émission stimulée peuvent survenir. S'il n'y a pas résonance, la plupart des interactions photon-électron sont élastiques, la direction et l'énergie du photon incident restent inchangées. Cependant l'interaction entre le photon et le "nuage" électronique ralentit le photon d'une quantité caractérisće par l'indice de réfraction du milieu condensé.

Si le cristal ne présente pas de centre de symétrie (le cristal est alors appelé noncentrosymétrique ou biréfringent) et est soumis à un champ électrique, il devient polarisé. L'interaction entre les photons incidents et le nuage électronique est affecté par les chargements induits par le champ électrique dans le nuage électronique. Macroscopiquement l'indice de réfraction du cristal évolue, les propriétés optiques du cristal ( $\mathrm{LiNbO}_{3}$ par exemple) s'en trouvent considérablement perturbées ce qui conduit à l'effet sctrooptique, dit de Pockels, utilisé pour déclencher les lasers (Q-Switch). 
Par analogie si un cristal polarisable est illuminé par un faisccau laser intense el cohérent à une fréquence wo, le fort champ électrique oscillant du faisceau laser va provoquer des oscillations dans le nuage électronique à la même fréquence $\omega_{0}$. Si un photon de fréquence $\omega_{p}$ interagit alors avec ce champ oscillant, il y a une probabilité finie d'interaction qui modifie la fréquence du photon $\omega_{\mathrm{p}}$ en une fréquence $\omega_{\mathrm{N}}$ telle que (Equation de maxwell)

$$
\omega_{N}=\omega_{0} \pm \omega_{p}
$$

Cette équaticia est la base du mélange de fréquences $\left(\omega_{\mathrm{p}} \neq \omega_{0}\right)$ ou du doublage de fréquence $\left(\omega_{\mathrm{p}}=\omega_{0}\right)$ des faisceaux laser. En effet si un faisceau laser monofréquence wo de forte intensité traverse le cristal la probabilité de produire un faisceau de fréquence $2 \omega_{0}$ est importante.

Plusieurs paramètres déterminent si la génération du second harmonique est efficace. Le cristal doit présenter une polarisabilité correcte et de bonnes propriétés optiques et le faisceau laser doit être suffisament énergique.

Comme la polarisation du cristal est directement fonction de l'intensité du faisceau laser incident, l'intensité du faisceau doublé est proportionnel au carré de l'intensité du faisceau fondamental (Tableau I). Ceci explique que le doublage de fréquence est généralement réalisé à partir de lasers déclenchés pour lesquels l'énergie délivrée est particulièrement importante.

Tableau I : Comparaison des rendements de conversion d'un laser YAG:Nd pulsé par du BBO et du KD*P [1]

\begin{tabular}{|c|c|c|c|c|}
\hline $\begin{array}{c}\text { Densité de Puissance } \\
\text { (MW.cm-2) }\end{array}$ & Puissance moyerne $(\mathrm{W})$ & $\begin{array}{c}\text { Taux de répétition } \\
\text { (pps) }\end{array}$ & Rendement BBO (\%) & $\begin{array}{c}\text { Rendement } K D \star P \\
(\mathscr{X})\end{array}$ \\
\hline $32^{\mathrm{a}}$ & 1,5 & 10 & 20 & 15 \\
\hline $87^{\mathrm{a}}$ & 4 & & 42 & 20 \\
\hline $125^{b}$ & 4,8 & & 43 & 37 \\
\hline $192^{b}$ & 4,5 & & 47 & 42 \\
\hline $167^{\mathrm{b}}$ & 8,5 & 20 & 38 & 20 \\
\hline $147^{\mathrm{c}}$ & 11,2 & 30 & 39 & 35 \\
\hline $125^{\mathrm{c}}$ & 17,5 & 50 & 30 & 28 \\
\hline $253^{\mathrm{c}}$ & 36 & & 37 & 35 \\
\hline & & & & \\
\hline
\end{tabular}

\section{2 Accord de phase}

La génération d'harmonique n'est pas un processus très efficace. Pour obtenir des faisceaux doublés d'intensité appréciable, des cristaux d'une longueur avoisinant $10 \mathrm{~mm}$ doivent être utilisés. Comme l'indice de réfraction d'un matériau varie avec la longueur d'onde, deux faisceaux de fréquence $\omega$ et $2 \omega$ ne se propagent pas à la même vitesse, leur phase relative change au fur et à mesure qu'ils se propagent dans le cristal. Les photons "doublés" en un point du cristal ne sont pas en phase avec ceux produits en d'autres points de l'échantillon. Il en résulte une interférence entre les différents faisceaux doublés qui minimise voire annule l'intensité du faisceau doublé en sortie de cristal. 
Le doublage de fréquence d'un faisccau laser ne sera efficace que si les vitesses de phase des ondes fondamentales et harmoniques sont identiques : accord de phase.

Considérons un cristal uniaxe, ce qui est le type de cristal le plus communément utilisé pour le doublage de fréquence. Ce cristal est caractérisé par deux indices de réfraction : l'ordinaire no et l'extraordinaire ne selon que la polarisation de lumière soit respectivement perpendiculaire et parallèle à l'axe optique.

Si le faisceau incident fait un angle $\theta$ avec l'axe optique, l'indice de réfraction pour l'onde extraordinaire est :

$$
\mathrm{ne}(\theta)=\mathrm{none} /\left(\mathrm{no}^{2} \sin ^{2} \theta+\mathrm{ne}^{2} \cos ^{2} \theta\right)^{1 / 2}
$$

et décrit une éllipsoïde en fonction de $\theta$ alors que l'indice de réfraction pour l'onde ordinaire est constant et égal à no et décrit une sphère en fonction de $\theta$.

Comme les indices de réfraction varient avec la longueur d'onde, si le cristal est suffisament biréfringent, l'ellipsoïde $\mathrm{ne}^{2 \omega}(\theta)$ de tharmonique $2 \omega$ et la sphère $\mathrm{no}^{\omega}(\theta)$ du fondamental peuvent s'intercepter selon un cercle. L'angle $\theta \mathrm{m}$ pour lesquels $\mathrm{ne}^{2 \omega}(\theta)$ et now $(\theta)$ se croisent correspond à un accord de phase de type I pour lequel les vitesses de phase des ondes fondamental et harmonique sont identiques.

Le cristal orienté selon l'angle $6 \mathrm{~m}$ aura alors un rendement de conversion de la fréquence $\omega$ important. Plusieurs types d'accord de phase sont possibles et il convient d'utiliser l'accord de phase de type I ou II selon la polarisation du faisceau incident (Tableau II).

Ainsi un même cristal peut générer différents harmoniques d'un faisceau fondamental. Il convient alors d'orienter le cristal selon l'angle $\theta \mathrm{m}$ de l'accord de phase considéré (Tableau III)

Tableau II : Conditions générales d'accord de phase d'un cristal uniaxe

\begin{tabular}{|c|c|c|c|}
\hline & tal uniaxe & Type I & Type II \\
\hline positif & ne $>$ no & $n o^{2 \omega}=n e^{\omega}(\theta m)$ & $\mathrm{no}^{2 \omega}=\left[\mathrm{ne}^{\omega}(\theta \mathrm{m})+\mathrm{no}^{\omega}\right] / 2$ \\
\hline négatif & ne $<$ no & $n e^{2 \omega}(\theta m)=n o^{\omega}$ & $n \mathrm{e}^{2 \omega}(\theta \mathrm{m})=\left[\mathrm{ne}^{\omega}(\theta \mathrm{m})+\mathrm{no}^{\omega}\right] / 2$ \\
\hline
\end{tabular}

Tableau III : Valeurs expérimentales des angles d'accord de phase pour le doublage de la fréquence $\lambda 1$ dans le $B B O[2]$

\begin{tabular}{|c|c|c|c|c|c|c|c|}
\hline $\begin{array}{c}\lambda_{1}(1 / \omega) \\
(\mathrm{nm})\end{array}$ & $\begin{array}{c}\lambda_{2}(1 / 2 \omega) \\
(\mathrm{nm})\end{array}$ & $\theta\left({ }^{\circ}\right)$ & Type & $\begin{array}{c}\lambda_{1}(1 / \omega) \\
(\mathrm{nm})\end{array}$ & $\begin{array}{c}\lambda_{2}(1 / 2 \omega) \\
(\mathrm{nm})\end{array}$ & $\theta\left({ }^{\circ}\right)$ & Type \\
\hline 1064,2 & 532,1 & 22,8 & I & 600 & 300 & 40 & I \\
\hline & & 32,7 & II & 582 & 291 & 42 & I \\
\hline & & & & 580 & 290 & 41 & I \\
\hline 780 & 390 & 29,9 & I & 532 & 266 & 48 & I \\
\hline 730 & 365 & 32,1 & I & & & 81 & II \\
\hline 709,4 & 354,7 & 33 & I & 529 & 264,5 & 50 & I \\
\hline & & 48 & II & 510 & 255 & 50,6 & I \\
\hline 694,3 & 347,1 & 36 & I & 488 & 244 & 55 & I \\
\hline 630 & 315 & 37 & I & 454 & 277 & 65 & I \\
\hline 616 & 308 & 39 & I & 411,6 & 205,8 & 83,1 & I \\
\hline
\end{tabular}


Néanmois, dans le cas d'un cristal uniaxe, si $\theta \mathrm{m}$ est différent de $90^{\circ}$, la direction des flux de puissance des faisceaux fondamental et harmonique (vecteurs de Poynting) ne coïncident pas parfaitement. Un petit angle dit de "walk off" sépare ces deux directions et limite ainsi le volume (ou la longueur) effectif du cristal utilisé pour le doublage de fréquence et diminue d'autant le rendement de conversion.

Comme les indices de réfraction d'un matériau varient avec sa température il est possible d'obtenir, pour certains cristaux, un accord de phase en conservant $\theta \mathrm{m}$ égal à $90^{\circ}$ mais en ajustant la température du cristal à une température où les conditions d'accord de phase sont respectées (Figure 1). L'accord de phase est alors appelé accord de phase non critique et correspond à un processus de conversion de fréquence particulièrement efficace où la longueur du cristal n'est pas limitée.

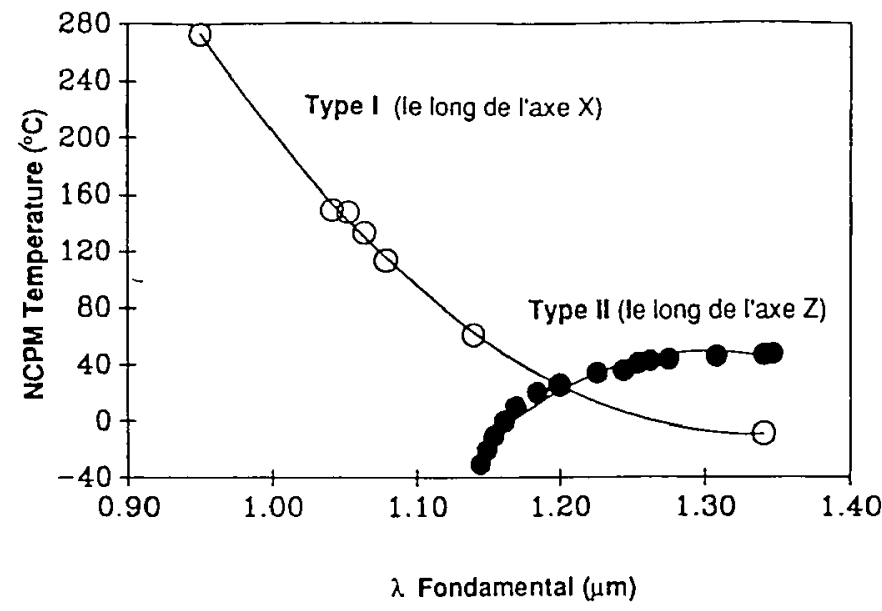

Figure 1 : Température du LBO permettant l'accord de phase non critique dans le doublage de fréquence du laser YAG:Nd [3]

\section{SELECTION DES MATERIAUX NON LINEAIRES}

En préambule à ce chapitre il parait utile de signaler qu'il n'existe pas de matériau non linéaire idéal. En fait selon les applications envisagées un couple laser-cristal doubleur est mieux adapté que d'autres.

Une conversion fortement efficace dépend de paramètres associés simultanément à la source laser et au cristal non linéaire. Pour la source les paramètres importants sont : la densité de puissance, la divergence du faisceau (Tableau D) et la longueur spectrale.

Les conditions requises pour le choix d'un matériau non linéaire sont : une biréfringence élevée (qui détermine son efficacité), une plage de transparence et d'accordabilité en phase étendue, un seuil de dommage optique élevé, une bonne stabilité thermique et chimique (hygroscopie et déliquescence éventuelles), une facilité d'élaboration sous forme monocristalline (qui conditionne les dimensions utiles du cristal et l'homogéneité des propriétés optiques), l'angle de "walk off", les acceptances thermique e1 angulaire.

L'élaboration des cristaux non linéaires conditionne fortement leur utilisation. En cffet, il faut que les dimensions des cristaux préparés soient suffisament grandes pour découper et polir des échantillons d'une longueur proche de $10 \mathrm{~mm}$ orientés pour l'accord de phase désiré et que la qualité optique de ces monocristaux soit bonne pour accéder à un rendement de conversion élevé. Les défauts éventuels du monocristal (occlusions gazeuses, inclusions de phases parasites, absorption résiduelle...) diminuent fortement le seuil de dommage optique. 


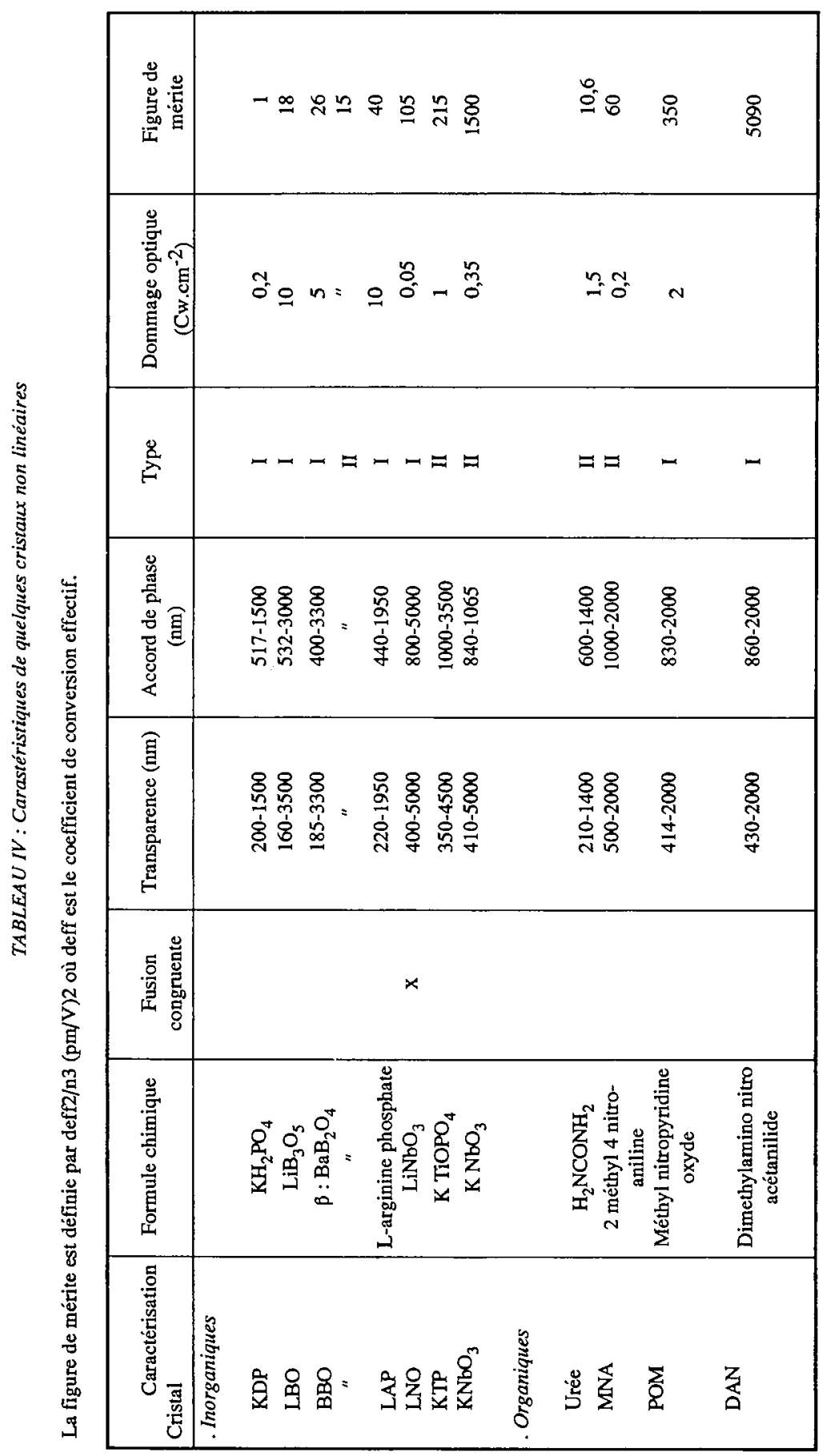


Si la composition des phases liquide et solide dun composé sont identiques et si ce matériau ne présente pas de transition de phase entre sa température de fusion et la température ambiante, ce composé, dit à fusion congruente, peut être élaboré sous forme de gros monocristaux par les techniques classiques de cristallogénèse à partir du bain fondu utilisées pour le silicium, le YAG:Nd... (Czochralski, Bridgman...). La qualité optique de ces cristaux est excellente et très homogène. Parmi les cristaux doubleurs inorganiques seul $\mathrm{LiNbO}_{3}$ présente une fusion congruente mais son seuil de dommage optique est particulièrement faible ce qui limite son utilisation (Tableau IV).Tous les autres composés inorganiques regroupés dans le tableau IV ne fondent pas de manière congruente, il convient alors d'utiliser un solvant pour diminuer la température du mélange solvant-matériau non linéaire au dessous de la température de décomposition ou de transition de phase du matériau et de ne cristalliser, par une diminution lente et progressive de la température du mélange $\left(\Delta \mathrm{T}<0,1^{\circ} \mathrm{C} \cdot \mathrm{h}^{-1}\right)$, que le matériau non linéaire. De telles techniques, dites du flux, certes améliorées depuis quelques années pour produire des cristaux plus gros : "Top Seeding Solution Growth" (TSSG), conduisent à la préparation de monocristaux dont la qualité optique n'est pas toujours excellente et homogène. En particulier les inclusions de flux diminuent considérablement le volume utile du monocristal.

Les matériaux organiques ont un rendement de conversion particulièrement élevé, cependant leur faible seuil de dommage optique et leur plage de transparence relativement restreinte dans l'ultraviolet limitent leur utilisation pour la génération d'harmonique dans l'ultraviolet (Tableau IV).

Parmi les matériaux inorganiques seuls le KDP, le LAP, le BBO et LBO présente une phase de transparence intéressante dans IUV. Le KDP a un pouvoir non linéaire et un seuil de dommage optique faible. Il est par conséquent assez délicat de délivrer un faisceau laser UV de forte intensité par conversion de fréquence dans le KDP. Les propriétés optiques du LAP paraissent très intéressantes dans l'ultraviolet. Toutefois le développement de ce cristal débuté vers 1984 s'est interrompu depuis. En revanche le développement de la cristallogénèse du BBO et du LBO, dont les propriétés sont particulièrement bien adaptées dans lUV, est en plein essor.

\section{BBO et LBO}

Ces deux cristaux ont été découverts par le Fujian Institute (Chine) en 1985 (BBO) et en 1989 (LBO) et sont commercialisés.

\section{IV. $1 \mathrm{BBO}$}

Le métaborate de baryum $\beta: \mathrm{BaB} 2 \mathrm{O} 4(\mathrm{BBO})$ est un cristal uniaxe de structure trigonale qui fond sans se décomposer mais présente une transition de phase autour de $925^{\circ} \mathrm{C}$. Seule la phase basse température $\beta$ est noncentrosymétrique et utile pour la conversion de fréquence. L'oxyde de sodium $\mathrm{Na} 2 \mathrm{O}$ ou/et le borate de sodium $\mathrm{NaBO} 2$ sont utilisés pour élaborer des monocristaux de $\mathrm{BBO}$ à une température inférieure à $925^{\circ} \mathrm{C}$. La taille maximale des cristaux élaborés par TSSG est $\varnothing=120 \mathrm{~mm}, \mathrm{~h}=15-20 \mathrm{~mm}$.

Les variations des indices de réfraction du BBO en fonction de la longueur d'onde sont décrites par :

$$
\begin{aligned}
& \mathrm{no}^{2}=2,7405-0,0155 \lambda^{2}+0,0184 /\left(\lambda^{2}-0,0179\right) \\
& \mathrm{no}^{2}=2,3730-0,0044 \lambda^{2}+0,0128 /\left(\lambda^{2}-0,0156\right)(\lambda \text { en } \mu \mathrm{m})
\end{aligned}
$$




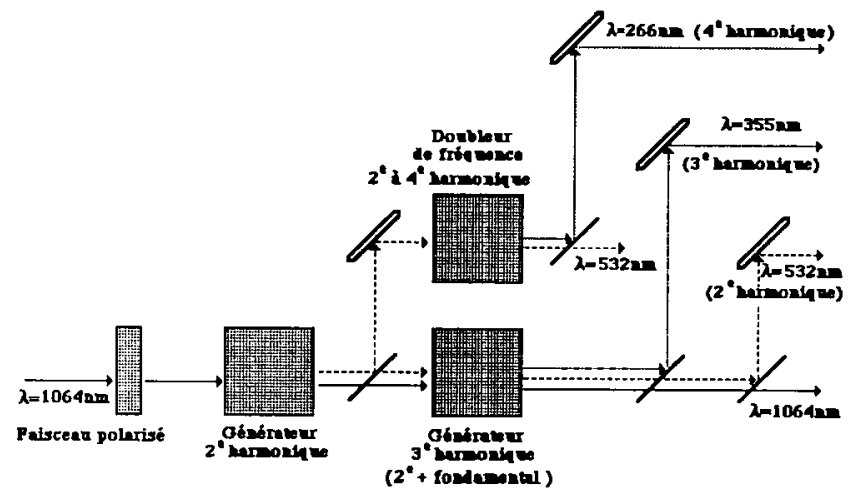

Figure 2 : Méthode de génération d'harmoniques d̀ partir d'un seul laser

En utilisant différents cristaux de BBO, il est possible d'obtenir jusqu'au $5^{\circ}$ harmonique d'un laser YAG:Nd à l'aide du montage décrit sur la figure 2. Les performances du BBO sont regroupées dans le tableau V.

Tableau V : Energies et rendements de conversion des harmoniques d 'un YAG:Nd dans du BBO [1]

\begin{tabular}{|c|c|c|c|c|c|c|c|c|}
\hline \multirow{2}{*}{$\begin{array}{c}\text { Fondamental } \\
(1064 \mathrm{~nm})\end{array}$} & \multicolumn{2}{|c|}{$2 \omega: 532 \mathrm{~nm}$} & \multicolumn{2}{|c|}{$3 \omega: 355 \mathrm{~nm}$} & \multicolumn{2}{|c|}{$4 \omega: 266 \mathrm{~nm}$} & \multicolumn{2}{c|}{$5 \omega=213 \mathrm{~nm}$} \\
\cline { 2 - 8 }$(\mathrm{mJ})$ & $(\mathrm{mJ})$ & $\%$ & $(\mathrm{~mJ})$ & $\%$ & $(\mathrm{~mJ})$ & $\%$ & $(\mathrm{~mJ})$ & $\%$ \\
\hline 220 & 105 & 48 & 39 & 18 & 18,5 & 8,4 & 5 & 2,3 \\
\hline 600 & 350 & 58 & 140 & 23 & 70 & 11,6 & 20 & 3,3 \\
\hline
\end{tabular}

De la même manière le BBO peut être utilisé pour doubler et tripler un laser alexandrite (730-780 nm), des implusions de $15 \mathrm{~mJ}$ sont ainsi produites entre 240 et $260 \mathrm{~nm}$. Un laser argon ionisé doublé par un cristal de BBO permet l'obtention de 36 raies UV dont la plus intense à $250,4 \mathrm{~nm}$ délivre $33 \mathrm{~mW}$. Le mélange de fréquence dans un cristal de $\mathrm{BBO}$ d'un laser $\mathrm{Al}_{2} \mathrm{O}_{3}: \mathrm{Ti}(790 \mathrm{~nm})$ de $700 \mathrm{~mW}$ et d'un laser $\mathrm{Ar}^{+}$doublé (257 $\mathrm{nm}$ ) de $15 \mathrm{~mW}$ délivre un faisceau accordable de 189 à $197 \mathrm{~nm}$ d'une puissance proche de quelques dizaines de nanowatts.

\section{2 LBO}

Le triborate de lithium $\mathrm{LiB}_{3} \mathrm{O}_{5}(\mathrm{LBO})$ est un cristal biaxe de structure orthorhombique qui se décompose avant sa fusion. Les cristaux sont élaborés par TSSG à partir d'un mélange de $\mathrm{Li}_{2} \mathrm{O}$ et de $\mathrm{B}_{2} \mathrm{O}_{3}$ de composition proche de $\mathrm{LiB}_{3} \mathrm{O}_{5}$. La qualité optique des cristaux est, par conséquent, très bonne car on ne travaille que dans le diagramme de phases binaire $\mathrm{Li}_{2} \mathrm{O}-\mathrm{B}_{2} \mathrm{O}_{3}$ : les inclusions de flux sont peu nombreuses. La taille maximale des cristaux élaborés par TSSG est $30 \times 30 \times 15 \mathrm{~mm}^{3}$. 
Les indices de réfraction sont décrits par les équations de Sellmeier suivantes :

$$
\begin{aligned}
& \mathrm{nx}^{2}=2,4532-0,0112 \lambda^{2}+0,0115 /\left(\lambda^{2}-0,0106\right) \\
& \mathrm{ny}^{2}=2,53969-0,0203 \lambda^{2}+0,0125 /\left(\lambda^{2}-0,0134\right) \\
& \mathrm{nz}^{2}=2,58515-0,0185 \lambda^{2}+0,0141 /\left(\lambda^{2}-0,0047\right)(\lambda \text { en } \mu \mathrm{m})
\end{aligned}
$$

Le LBO est le matériau dont le seuil de dommage optique est le plus élevé. La génération du second et du troisième harmonique d'un laser YAG:Nd très puissant conduit à l'obtention de longueurs d'onde à 532 et $355 \mathrm{~nm}$ avec des rendements de conversion respectifs de 70 et de $60 \%$. Le doublage de fréquence d'un laser $\mathrm{Al}_{2} \mathrm{O}_{3}: \mathrm{Ti}$ conduit à la réalisation d'un laser à $400 \mathrm{~nm}$ d'une puissance de $250 \mathrm{~mW}$, soit un rendement de l'ordre de $20 \%$.

Par ailleurs le mélange des fréquences d'un laser YAG:Nd et d'un laser à colorant doublé dans un cristal du LBO permet l'obtention d'un faisceau laser à $232,5 \mathrm{~nm}$ avec un rendement de l'ordre de $24 \%$.

\section{CONCLUSIQN}

Parmi les nombreux matériaux non linéaires étudiés le BBO et LBO semblent les mieux adaptés à la génération d'harmoniques dans l'ultraviolet.

Le BBO est un matériau mature dont la forte biréfringence permet la génération d'harmoniques du YAG:Nd (jusqu'au 5ème) avec un rendement de conversion important.. Toutefois le rendement de conversion du BBO est très sensible à la qualité du faisceau et à l'angle d'incidence du laser. Ainsi le BBO n'est généralement pas bien approprié à la génération d'harmoniques de lasers de grande divergence et de mauvaise qualité en termes de modes.

Le LBO est un nouveau cristal surtout étudié jusquaa présent pour ses applications dans le doublage du YAG:Nd. Le balayage en température et en angle font apparaitre de nombreuses applications en doublage et en triplage des lasers YAG:Nd surtout si l'on tient compte de la qualité optique des cristaux obtenus jusqu'alors et de son seuil de dommage optique particulièrement élevé. En revanche sa plage d'accord de phase relativement réduite limite ses applications dans l'ultraviolet.

\section{REEERENCES}

[1] R.S. Adhav, S.R. Adhav et al. Laser Focus, Sept. 87, 88

[2] D.N. Nikogosyan - App. Phys. A52 (91) 359

[3] J. Ling, Z. Sun et al. Opto 64 (91) 40 